\title{
A nomogram for predicting the HER2 status in female patients with breast cancer in China: a nationwide, multicenter, 10-year epidemiological study
}

Huimin Zhang ${ }^{1}$, Peiling Xie ${ }^{1}$, Zhuoying Li ${ }^{1}$, Rong Huang ${ }^{2,3}$, Weiliang Feng ${ }^{4}$, Yanan Kong ${ }^{5}$, Feng X X ${ }^{6}$, Lin Zhao ${ }^{7}$, Qingkun Song ${ }^{2}$, Jing $\mathrm{Li}^{2}$, Baoning Zhang ${ }^{8}$, Jinhu Fan², Youlin Qiao ${ }^{2}$, Xiaoming Xie ${ }^{5}$, Shan Zheng ${ }^{9}$, Jianjun $\mathrm{He}^{{ }^{*}}$ and Ke Wang ${ }^{1 *}$

\begin{abstract}
Background: The concordance rate of human epidermal growth factor receptor 2 (HER2) status between core needle biopsy (CNB) and subsequent excisional biopsies of the same tumor varies from 81 to 96\%, which may cause inappropriate neoadjuvant therapy that impair the potential benefit from HER2 targeted therapy for patients. This study aimed to establish a nomogram to predict the HER2 status pre-operatively as an auxiliary diagnosis to CNB assessment.

Methods: Among 4211 breast cancer patients cataloged in the Nation-wide Multicenter 10-year Retrospective Clinical Epidemiological Study of Breast Cancer in China, 2291 patients with complete relevant information were included in this study, which were further randomized 3:1 and divided into a training set and a validation set. The nomogram was established based on independent predictors of HER2 positivity recognized by logistic regression analysis and further validated internally and externally.

Results: The multivariate logistic regression analysis showed that T-stage, N-stage, estrogen receptor (ER) status, progesterone receptor (PR) status were independent predictors for HER2 status. The nomogram was thereby constructed by those independent predictors as well as histology type. The areas under the receiver operating characteristic curve (AUC) of the training set and the validation set were 0.636 and 0.681 , respectively. The calibration plots demonstrated good fitness of the nomogram for HER2 status prediction. With the optimal cutoff value, the nomogram yielded $80.0 \%$ sensitivity, $43.1 \%$ specificity in the training set and $81.1 \%$ sensitivity, $49.8 \%$ specificity in the validation set.
\end{abstract}

Conclusions: The present nomogram can provide valuable information on HER2 status and combined with standard CNB assessment, clinicians could make more appropriate decision on neoadjuvant therapy of breast cancer.

Keywords: Breast cancer, HER2 status, Nomogram, Prediction and validation

\footnotetext{
*Correspondence: chinahjj@163.com; 979915080@qq.com

1 Department of Breast Surgery, the First Affiliated Hospital of Xi'an Jiaotong

University, 277 West Yanta Road, 710061 Xi'an, People's Republic of China

Full list of author information is available at the end of the article
}

(c) The Author(s). 2019 Open Access This article is distributed under the terms of the Creative Commons Attribution 4.0 International License (http://creativecommons.org/licenses/by/4.0/), which permits unrestricted use, distribution, and reproduction in any medium, provided you give appropriate credit to the original author(s) and the source, provide a link to the Creative Commons license, and indicate if changes were made. The Creative Commons Public Domain Dedication waiver (http://creativecommons.org/publicdomain/zero/1.0/) applies to the data made available in this article, unless otherwise stated. 


\section{Background}

Breast cancer has been reported to be the most common malignant tumor and the leading cause of cancer death among women worldwide [1]. The utilization of neoadjuvant therapy (preoperative systemic therapy) provide the opportunity to monitor response during treatment, and help to increase the curative intervention as well as the breast conservation rate by reducing tumor burden [2]. The roles of CNB have been well established as an important preoperative diagnostic method for breast lesions. CNB is less invasive than excision biopsy and provide more information than fine needle aspiration (FNA). In the case of neoadjuvant therapy of breast cancer, CNB is the gold standard for pathological diagnosis and molecular subtype assessment.

Emerging data show that neoadjuvant chemotherapy combined with HER2-targeted therapy yields a higher pathologic complete response $(\mathrm{pCR})$ rate and favorable disease-free and overall survival when compared with neoadjuvant chemotherapy alone in women with HER2-positive breast cancers [3-8]. The increasing number of HER2 positive breast cancer patients being treated in the neoadjuvant setting gives rise to the need to accurately assess HER2 status on the CNB material that often is the only tissue available before treatment in these patients. However, due to intratumoral heterogeneity, the concordance rate of HER2 status between CNB and subsequent excisional biopsies of the same tumor varies from 81 to $96 \%$ as reported by different institutions [9-12].

In order to improve the accuracy of HER2 assessment in CNB specimens so that patients could get appropriate neoadjuvant therapy, we intend to identify possible predictors based on a nation-wide multicenter data spans 10 years in China and construct a nomogram for predicting HER2 status, which will increase the accuracy of HER2 assessment pre-operatively. In combination with $\mathrm{CNB}$ and nomogram prediction, it may provide more accurate information on whether the HER2 targeted therapy is needed in the neoadjuvant setting.

\section{Methods}

Study design and data collection

Data was obtained from the hospital-based, multicenter, 10-year (1999-2008), retrospective study of randomly selected pathology confirmed primary female breast cancer cases via medical chart review which was approved by the ethics committee of the Cancer

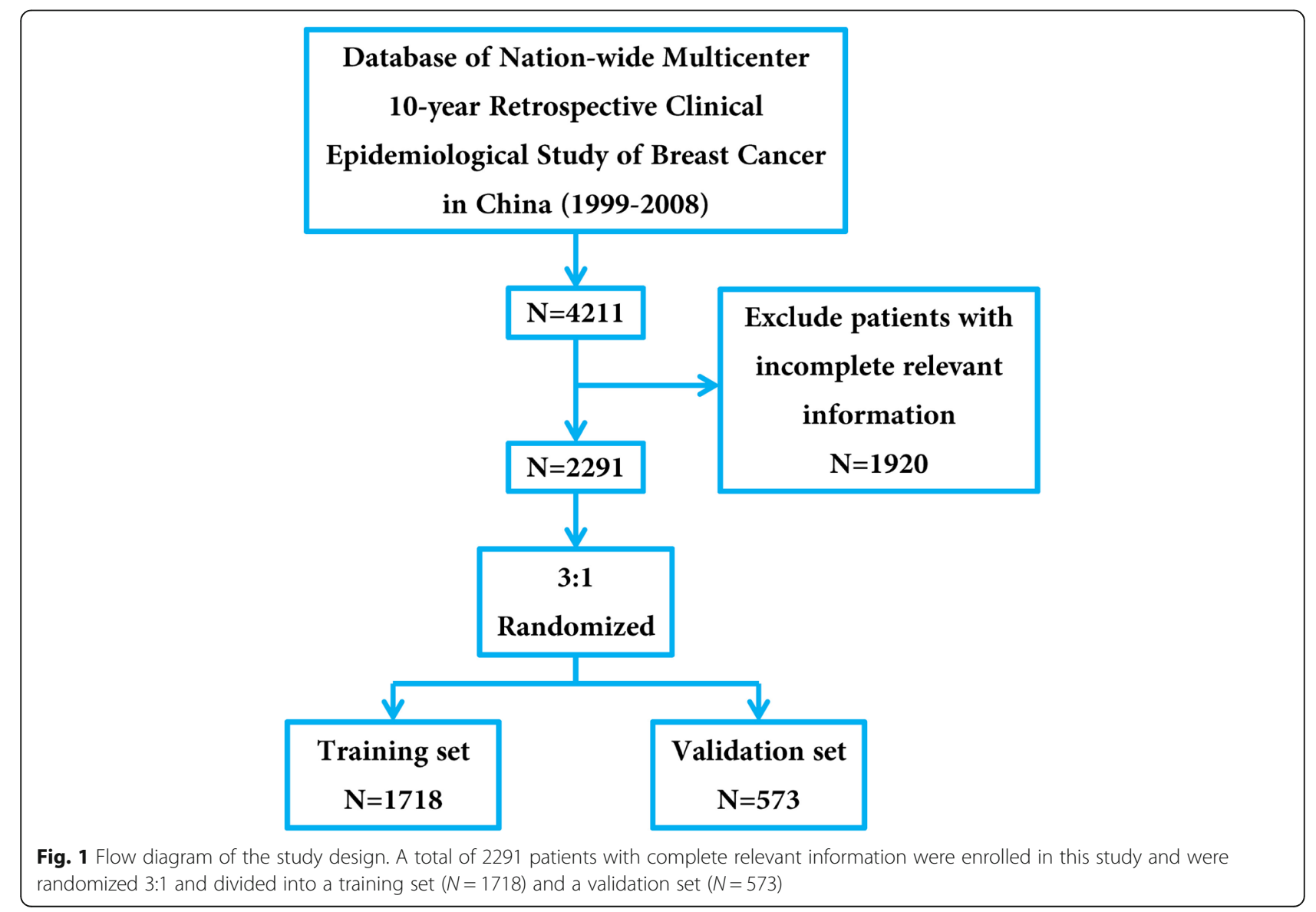


Foundation of China. In order to obtain the population, China was stratified into 7 geographic regions according to the traditional administrative district definition (north, northeast, northwest, middle, east, south and southwest); these regions extend over the majority of the country and represent different levels of breast cancer burden (Additional file 1: Figure S1) [13]. Of these patients, 4211 patients with a median age of 48 years (range: 21-86 years) were enrolled in this study. The age distribution of these patients conformed to 1999-2008 population-based breast cancer incidence data retrieved from the National Central Cancer Registry database (Additional file 1: Figure S2) [14]. One university hospital with good standard quality from each region was selected to provide the required study cases. Hospital records were reviewed by local clerks within each hospital according to the designated protocol. Further methodological details including patients selection, pathologic diagnostic criteria, data collection and quality control can be found in our previously published papers $[13,15]$.

Among overall population of 4211 patients, 2291 patients with complete relevant information (age, BMI, location of lesions, local infiltration, T-stage, N-stage, ER, PR, HER2 and histologic type) were included in this study, which were further randomized 3:1 and divided into a training set $(N=1718)$ and a validation set $(N=573)$.

In this study, IHC method was used to determine the HER2 status, scores of 0 and $1+$ be regarded as HER2-negative and that HER2 scores of $3+$ be considered as HER2-positive. HER2 score of $2+$ is regarded as HER2-borderline and HER2-borderline cases were excluded in our cohort due to low utilization rate of FISH assay during 1999-2008 in China.

\section{Nomogram construction and validation}

To develop a well-calibrated nomogram for predicting the probability of HER2 positivity, univariate as well as multivariate logistic regression analyses were performed to screen the predictors for HER2 positivity. Independent predictors $(P<0.05$ in the multivariate logistic regression analysis) as well as clinical significant predictors were included in the nomogram construction. The Hosmer and Lemeshow test was applied to assess goodness of fit of the model, and $P>0.05$ indicated a good fit. The odds ratios (ORs) and 95\% confidence intervals (CIs) were also calculated.

The nomogram was validated internally in the training set and externally in the validation set. A receiver-operating characteristic (ROC) curve was used to evaluate the effectiveness of the nomogram. Area under the curve (AUC) was calculated. The AUC ranged from 0 to 1 , with 1 indicating perfect concordance, 0.5 indicating no
Table 1 Clinicopathological characteristics of the study populations

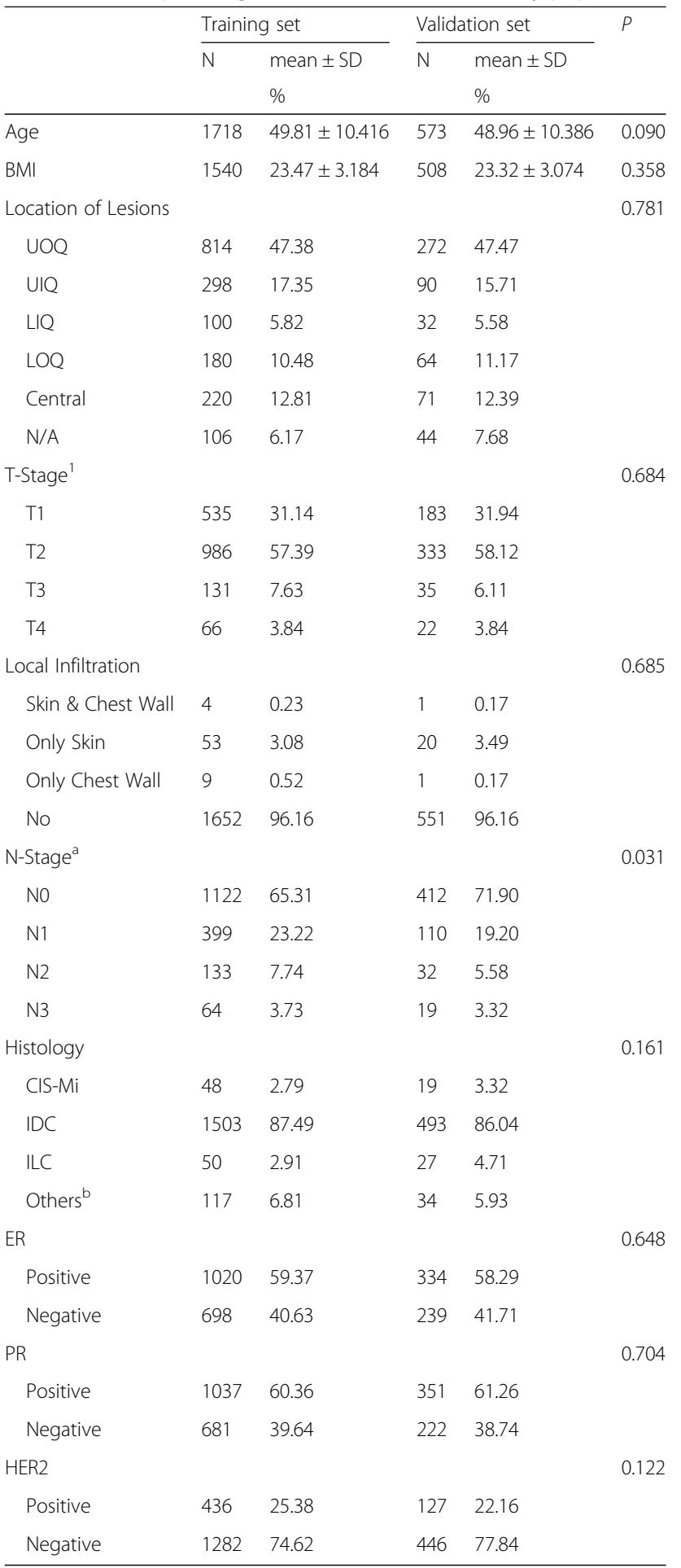

${ }^{\mathrm{a}} \mathrm{T}$-stage and $\mathrm{N}$-stage were both clinical stage determined by clinical (physical examination or radiologic) measurements

'Others: tubular carcinoma, mucinous carcinoma, medullary carcinoma Abbreviations: BMI body mass index, LIQ lower-inner quadrant, $L O Q$ lower-outer quadrant, UIQ upper-inner quadrant, UOQ upper-outer quadrant, N/A not available, CIS-Mi ductal/lobular carcinoma in situ and microinvasive carcinoma; IDC infiltrating ductal carcinoma, ILC infiltrating lobular carcinoma, ER estrogen receptor, $P R$ progesterone receptor, HER2 human epidermal growth factor receptor-2 
better than chance, and 0 indicating discordance. Statistical differences between different AUCs were investigated by the DeLong method. The calibration plot with bootstrapping was used to illustrate the association between the actual probability and the predicted probability.

All reported $P$ values are two-sided. The statistical analysis was carried out using SPSS (version 19.0; SPSS
Company, Chicago, IL) and $\mathrm{R}$ software version 3.4 (http://www.r-project.org).

\section{Results}

Clinicopathological features

There were 4211 breast cancer patients cataloged in the Nation-wide Multicenter 10-year Retrospective Clinical Epidemiological Study of Breast Cancer in China. After

Table 2 Analysis of risk factors for HER-2 positivity

\begin{tabular}{|c|c|c|c|c|}
\hline & \multicolumn{2}{|l|}{ Univariate analysis } & \multicolumn{2}{|l|}{ Multivariate analysis } \\
\hline & OR $(95 \% \mathrm{Cl})$ & P & OR $(95 \% \mathrm{Cl})$ & $P$ \\
\hline Age & $0.994(0.984-1.005)$ & 0.297 & & \\
\hline BMI & $0.995(0.960-1.031)$ & 0.769 & & \\
\hline \multicolumn{5}{|l|}{ Location of Lesions } \\
\hline UOQ & 1 & & & \\
\hline UIQ & $1.094(0.813-1.471)$ & 0.554 & & \\
\hline LIQ & $1.030(0.645-1.646)$ & 0.900 & & \\
\hline LOQ & $0.822(0.561-1.204)$ & 0.313 & & \\
\hline Central & $0.600(0.411-0.877)$ & 0.008 & & \\
\hline N/A & $1.100(0.701-1.725)$ & 0.679 & & \\
\hline \multicolumn{5}{|l|}{ T-Stage } \\
\hline $\mathrm{T} 1$ & 1 & & 1 & \\
\hline $\mathrm{T} 2$ & $0.787(0.621-0.998)$ & 0.048 & $0.720(0.562-0.921)$ & 0.009 \\
\hline T3 & $0.767(0.492-1.196)$ & 0.242 & $0.623(0.392-0.989)$ & 0.045 \\
\hline $\mathrm{T} 4$ & $0.607(0.322-1.145)$ & 0.123 & $0.493(0.256-0.949)$ & 0.034 \\
\hline \multicolumn{5}{|l|}{ Local Infiltration } \\
\hline No & 1 & & & \\
\hline Only Skin & $0.761(0.388-1.492)$ & 0.426 & & \\
\hline Only Chest Wall & $0.830(0.172-4.012)$ & 0.817 & & \\
\hline Skin \& Chest Wall & 0.000 & 0.999 & & \\
\hline \multicolumn{5}{|l|}{ N-Stage } \\
\hline No & 1 & & 1 & \\
\hline N1 & $1.165(0.895-1.517)$ & 0.256 & $1.172(0.892-1.540)$ & 0.255 \\
\hline N2 & $1.830(1.249-2.681)$ & 0.002 & $1.747(1.182-2.583)$ & 0.005 \\
\hline N3 & $2.605(1.559-4.351)$ & 0.000 & $2.866(1.683-4.879)$ & 0.000 \\
\hline \multicolumn{5}{|l|}{ Histology } \\
\hline CIS-Mi & 1 & & 1 & \\
\hline IDC & $0.990(0.518-1.890)$ & 0.975 & $0.919(0.475-1.777)$ & 0.801 \\
\hline $\mathrm{ILC}$ & $0.367(0.127-1.064)$ & 0.065 & $0.379(0.129-1.110)$ & 0.077 \\
\hline Others & $0.337(0.143-0.794)$ & 0.013 & $0.296(0.124-0.710)$ & 0.006 \\
\hline \multicolumn{5}{|l|}{ ER } \\
\hline Negative & 1 & & 1 & \\
\hline Positive & $0.591(0.475-0.736)$ & 0.000 & $0.690(0.522-0.914)$ & 0.010 \\
\hline \multicolumn{5}{|l|}{$P R$} \\
\hline Negative & 1 & & 1 & \\
\hline Positive & $0.602(0.483-0.750)$ & 0.000 & $0.726(0.548-0.961)$ & 0.025 \\
\hline
\end{tabular}


excluding 1920 patients with incomplete relevant information, 2291 eligible patients were included in the study which were randomized 3:1 and divided into a training set $(N=1718)$ and a validation set $(N=573)$ (Fig. 1$)$. The clinicopathological features were similar between the training set and the validation set (Table 1).

\section{Predictors for HER2 positive breast Cancer}

We used logistic regression as univariate and multivariate analysis to evaluate the risk factors for HER2 positive breast cancers in the training set. T-stage, $\mathrm{N}$-stage, ER and PR were risk factors for HER2 positive breast cancer in the univariate analysis, and these variables were further confirmed as independent risk factors in multivariate analysis (Table 2). According to the results, ER positive patients $(P=0.01$, OR $=0.690$ [95\% CI: $0.522-$ $0.914])$ and $\mathrm{PR}$ positive patients $(P=0.025$, OR $=0.726$ [95\% CI: 0.548-0.961]) were less likely to be HER2 positive than ER negative patients and PR negative patients, and patients with T2 $(P=0.009, \mathrm{OR}=0.720$ [95\% CI: $0.562-0.921])$, T3 $(P=0.045$, OR $=0.623$ [95\% CI: $0.392-$ $0.989])$ and $\mathrm{T} 4(P=0.034$, OR $=0.493$ [95\% CI: $0.256-$ 0.949]) stage were less likely to be HER2 positive compared with those with $\mathrm{T} 1$ stage. In contrast, patients of N2 $(P=0.005$, OR $=1.747$ [95\% CI: $1.182-2.583])$ and N3 $(P=0.000, \mathrm{OR}=2.866$ [95\% CI: $1.683-4.879])$ stage were more likely to have HER2 positive breast cancer than patients of N0 stage. Histology was not significantly associated with HER2 positivity.

\section{Construction and validation of the nomogram}

Based on the results of the multivariate analysis, a nomogram was constructed with independent predictors for HER2 status including T-stage, $\mathrm{N}$-stage, ER status and PR status as well as histology (Fig. 2). A total points is the sum of points for each variable (top plotting scale) and the probability of HER2 positivity is the corresponding number of the total points in the nomogram (bottom plotting scale). The $P$-value of the Hosmer and Lemeshow test was 0.055 , indicting a good statistical fit.

The ROC curve analysis was performed to validate the nomogram internally in the training set (Fig. 3a) and externally in the validation set (Fig. 3b). In the training set, the AUC was 0.636 (95\% CI: $0.607-0.665$ ). In the validation set, the AUC was 0.681 (95\% CI: 0.631-0.731). There's no statistical difference between two AUCs $(P=1)$. The calibration plot (Fig. 4) indicated that the nomogram was well calibrated.

As shown in Table 3, higher cutoff value resulted in the increasing of specificity and positive predictive value, while sensitivity and negative predictive value decreased. According to the Youden's method [16], optimal cutoff values of the training and validation sets were 0.212 (sensitivity: $80.0 \%$, specificity: $43.1 \%$, positive predictive value: $32.4 \%$, negative predictive value: $86.4 \%$ ) and 0.204 (sensitivity: $81.1 \%$, specificity: $49.8 \%$, positive predictive value: $31.5 \%$, negative predictive value: $90.2 \%)$, respectively (Table 4).

\section{The application of the nomogram}

To display the application of the nomogram, we took ten patients who were tested for HER2 status in tissues from both the core needle biopsy and the excision as examples (Table 5). Patients 1-4 have concordant HER2 status in CNB specimens, excisional specimens and nomogram prediction. Patients 5 and 6 were HER2

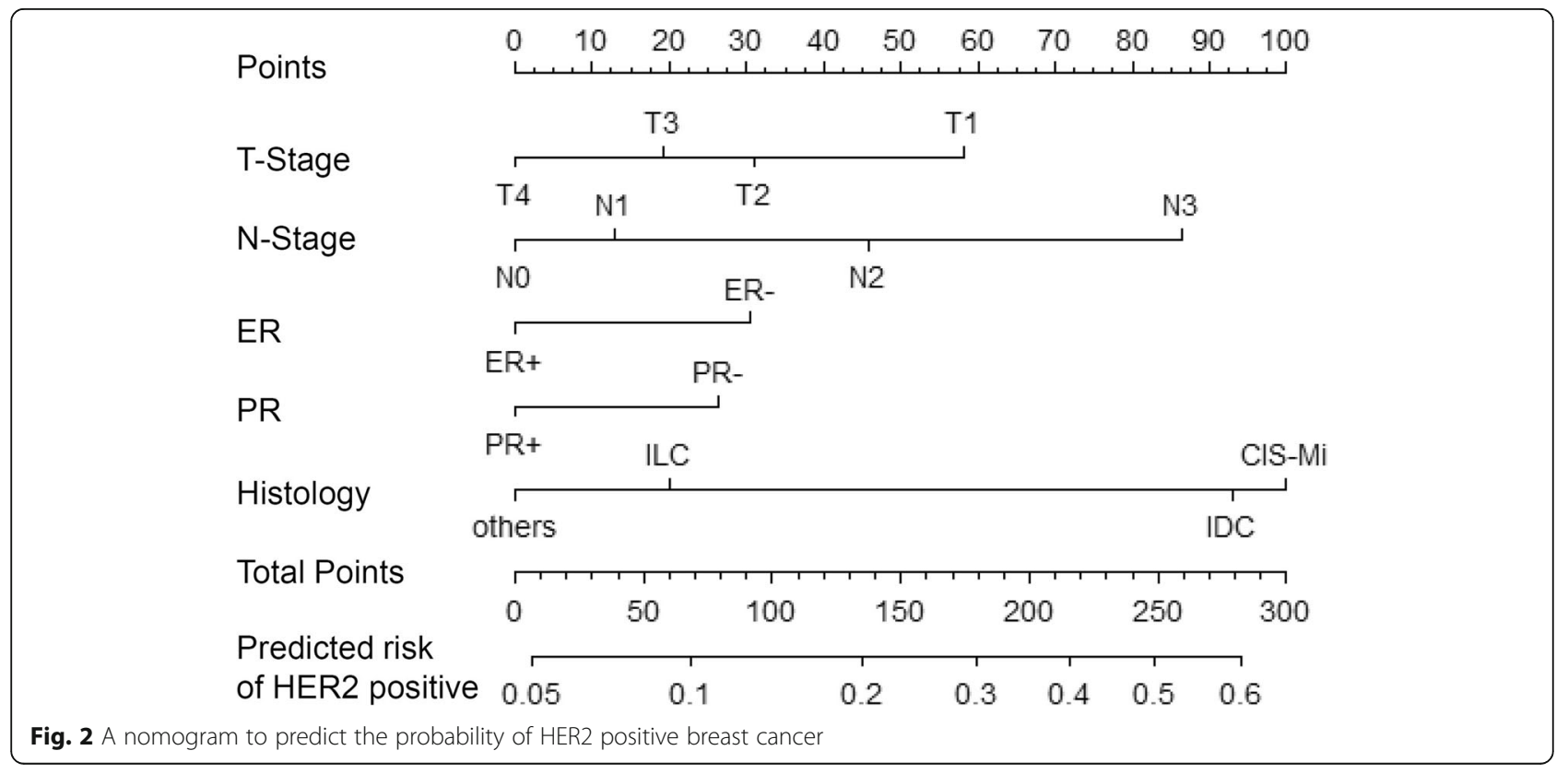




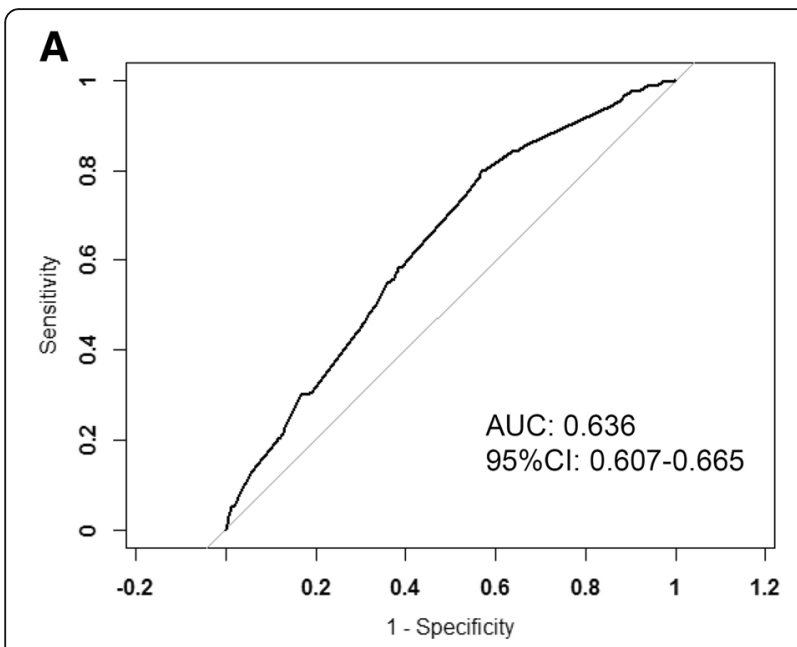

B

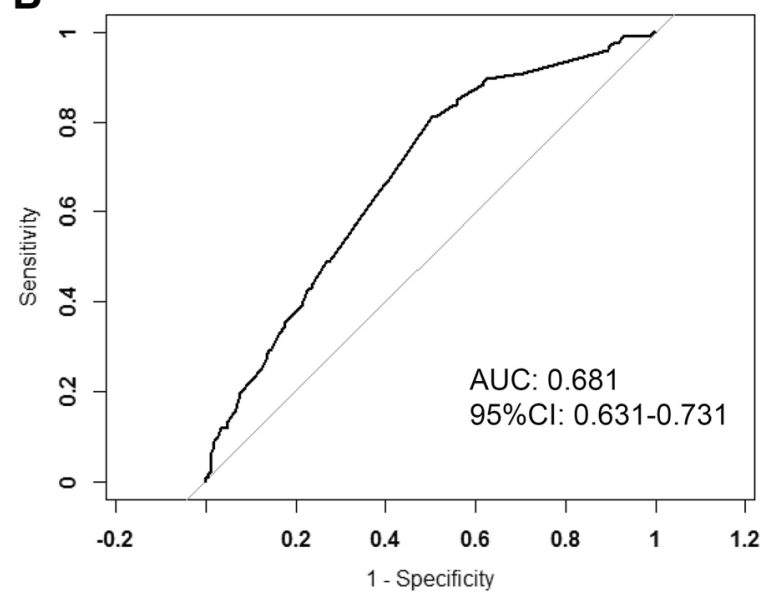

Fig. 3 Validation of the nomogram. (a) Internal validation using the ROC curve in the training set. The area under the ROC curve (AUC) is $0.636,95 \%$ confidence interval $(95 \% \mathrm{Cl}, 0.607-0.665)$. (b) External validation using ROC in the validation set. The AUC is 0.681 (95\% Cl, 0.631-0.731)

positive in the CNB specimens, but HER2 negative in the excisional specimens which were concordant with our prediction done by the nomogram. Patient 7 was HER2 negative in the CNB specimen, but HER2 positive in the excisional specimen which was concordant with our prediction done by the nomogram. Patients $8-10$ were HER2-borderline in the CNB specimens, by using the nomogram, prediction on HER2 status were consistent with the result in excisional specimens.

\section{Discussion}

In our current research, we first randomized the population containing 2291 patients with completed relevant information into a training set and a validation set. Based on the multivariate logistic regression analysis, we identified independent variables for predicting the HER2 status. Patients who were ER positive, PR positive were less likely to be HER2 positive than those who were ER negative, PR negative. Patients who were of T1 stage were more likely to be HER2 positive than those who were of T2, T3 and T4 stage. Patients with N2, N3 stage were more likely to be HER2 positive than those with N1 stage. Histology type did not show statistically significance in the multivariate logistic regression analysis. Next, we constructed a nomogram on the basis of these predictors as well as clinical significant predictors (histology). The AUCs in the training (internal validation) and validation sets (external validation) were 0.636 and 0.681 , respectively.

Tissue diagnosis of breast cancer by CNB before proceeding to any kind of treatment is the gold standard of modern medical practice according to clinical practice guidelines. The accuracy of histologic identification by $\mathrm{CNB}$ is superior to the cytological diagnosis by fine needle aspiration and $\mathrm{CNB}$ specimens could provide more tissue for further immunohistochemical (IHC) assessment if neoadjuvant therapy is indicated. Accordingly, physicians can discuss the treatment plan with the patient preoperatively and give her the chance of one-step operation or perform the neoadjuvant treatment based on the assessment of ER, PR, HER2 and Ki67 status in the CNB samples. In cases of pCR, this will be the only source for the evaluation of breast cancer molecular subtype. It can also determine the molecular subtype more accurately than the IHC exam of an excised tumor with partial response to neoadjuvant chemotherapy.

Data from clinical trials have shown that patients with HER2 positive breast carcinomas have significantly better responses (more frequently obtaining pathologic complete response and greater percent disease-free survival) when treated with HER2 targeted therapy simultaneously with neoadjuvant chemotherapy than with neoadjuvant chemotherapy alone [17]. With the increasing use of neoadjuvant therapies, clinicians require accurate information on HER2 status at the time of $\mathrm{CNB}$ as false negative HER2 result might impair the potential benefit from HER2 targeted therapy for patients. Concordance rate of HER2 status between CNB and subsequent excisional biopsies of the same tumor varies from 81 to $96 \%$ as reported by different institutions [9-12]. The disconcordance between $\mathrm{CNB}$ and excisional biopsy were mainly due to intratumoral heterogeneity. Studies showed that to improve the accuracy of HER2 status as well as other important information from $\mathrm{CNB}$, a minimum of four cores is required [18]. And when comparing 14-, 16-, 18-gauge needles, the accuracy rose with needles of increasing size [19]. These results suggested that diagnostic accuracy of $\mathrm{CNB}$ increased with the increase of harvested specimens. 


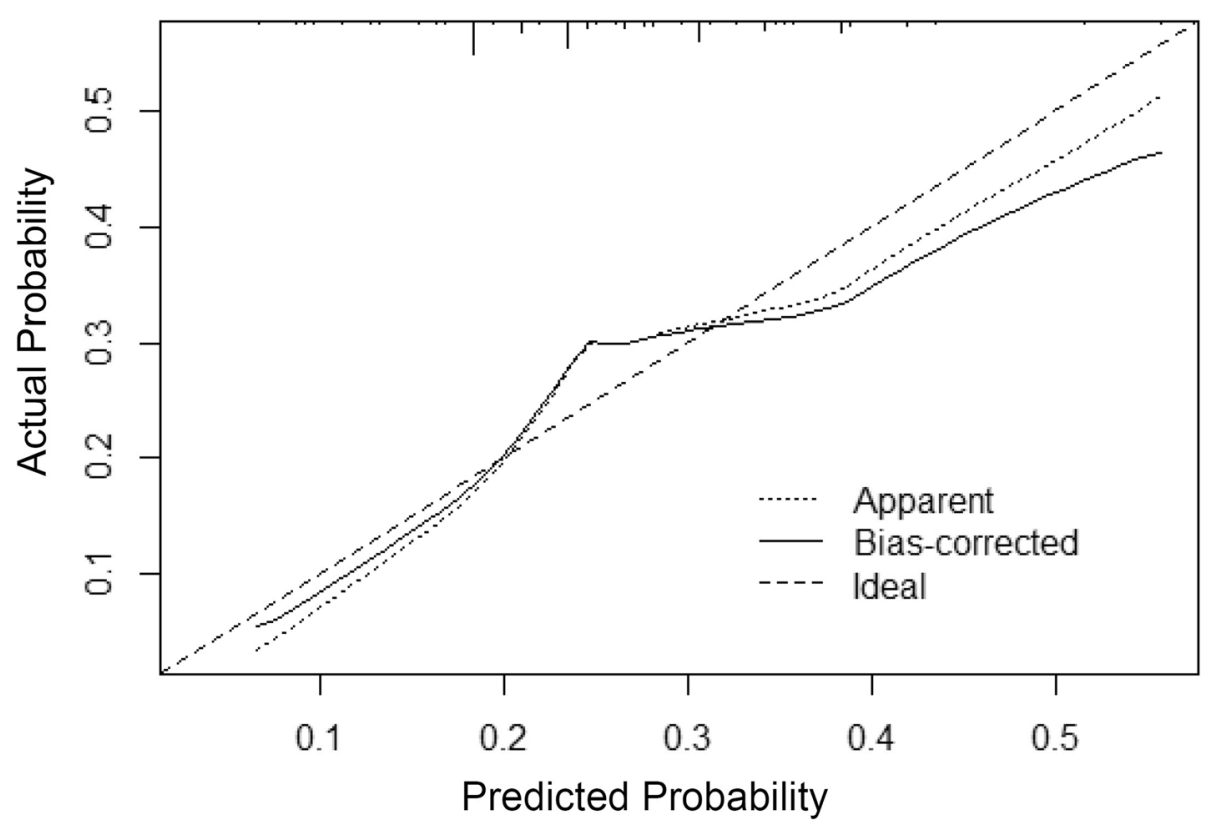

Fig. 4 Calibration plots of the nomogram for the probability of HER2 positive breast cancer (bootstrap 1000 repetitions)

However, improving the accuracy of HER2 status in CNB specimens by increasing the cores and needle size is invasive and may cause more complications after $\mathrm{CNB}$, so we developed noninvasive method - a nomogram to predict the HER2 status in the entire tumor prior to the surgery. If the HER2 status from CNB specimens is different from the nomogram predicted, then additional CNB may be needed to verify the exact status of HER2, in order to avoid inappropriate neoadjuvant therapy caused by false negative assessment of HER2.

In our study, we analyzed the common clinicopathological features including age, BMI, location of tumor, T-stage, local infiltration, $\mathrm{N}$-stage, histology type, ER expression and PR expression in HER2-positive and -negative cohort, and found that ER negative, PR negative, T1 stage and N2/N3 stage were independent risk factors for HER2 positive breast cancer. Traina et al. demonstrated that HER2 overexpression was significantly correlated with negative hormone receptor (HR) status, positive nodal status and G3 tumor grade based on data from
1355 Italian breast cancer patients [20]. Study from Morocco which included 1508 patients found that overexpression of HER2 was associated with high tumor grade, vascular space invasion and ER negativity significantly [21]. Another study from a single center in China suggested that ER status, PR status and tumor grade were significantly associated with HER2 status [22]. Different studies from different institution indicated the same finding that HER2 overexpression is correlated with HR negativity, while the relationship between HER2 expression and other clinicopathologic parameters varies, such as tumor dimension, nodal involvement and tumor grading.

The nomogram, a simple graphical prediction tool, allows oncologists to assess the predictive risk of individuals [23]. And it's been considered as an important component of modern medical decision making [24]. Another advantage of the nomogram is that it is noninvasive. So far, this is the first nomogram constructed to predict HER2 status based on nation-wide multicenter data in breast cancer. The nomogram we constructed

Table 3 Values of sensitivity, specificity, and predictive values of the predicted probability at different cutoff values

\begin{tabular}{|c|c|c|c|c|c|c|c|c|}
\hline \multirow{2}{*}{$\begin{array}{l}\text { Predicted } \\
\text { probability }\end{array}$} & \multicolumn{4}{|c|}{ Training set } & \multicolumn{4}{|c|}{ Validation set } \\
\hline & sensitivity & specificity & PPV & NPV & sensitivity & specificity & PPV & NPV \\
\hline$\geq 0.1$ & $98.9 \%$ & $4.8 \%$ & $26.1 \%$ & $92.5 \%$ & $98.4 \%$ & $4.5 \%$ & $22.7 \%$ & $90.9 \%$ \\
\hline$\geq 0.2$ & $83.9 \%$ & $36.5 \%$ & $31.0 \%$ & $87.0 \%$ & $83.5 \%$ & $34.3 \%$ & $26.6 \%$ & $87.9 \%$ \\
\hline$\geq 0.3$ & $44.0 \%$ & $70.8 \%$ & $33.9 \%$ & $78.8 \%$ & $43.3 \%$ & $71.7 \%$ & $30.4 \%$ & $81.6 \%$ \\
\hline$\geq 0.4$ & $9.6 \%$ & $95.9 \%$ & $44.2 \%$ & $75.7 \%$ & $10.2 \%$ & $97.5 \%$ & $54.2 \%$ & $79.2 \%$ \\
\hline$\geq 0.5$ & $4.8 \%$ & $98.7 \%$ & $53.8 \%$ & $75.3 \%$ & $4.7 \%$ & $99.5 \%$ & $66.7 \%$ & $78.5 \%$ \\
\hline
\end{tabular}

NPV negative predictive value, $P P V$ positive predictive value 
Table 4 Values of sensitivity, specificity, and predictive values of the predicted probability at the optimal cutoff value

\begin{tabular}{llllll}
\hline & The Optimal Cutoff & Sensitivity & Specificity & PPV & NPV \\
\hline Training set & 0.212 & $80.0 \%$ & $43.1 \%$ & $32.4 \%$ & $86.4 \%$ \\
Validation set & 0.204 & $81.1 \%$ & $49.8 \%$ & $31.5 \%$ & $90.2 \%$
\end{tabular}

NPV negative predictive value, $P P V$ positive predictive value

*The optimal cutoff value is determined according to the Youden's method

here is not aimed to replace the molecular test of HER2, but to prevent the false negative of HER2 due to CNB sample limitation. According to the Youden's method [16], the optimal cutoff value of the training and validation set in this study were 0.212 and 0.204 , at which the sensitivity were 80 and $81.1 \%$ while the specificity were only 43.1 and $49.8 \%$, respectively. As we can't have both high sensitivity and high specificity, higher sensitivity is what we more needed considering that the purpose of our nomogram is to prevent false negativity. By using

Table 5 The application of the nomogram

\begin{tabular}{|c|c|c|c|c|c|c|c|c|c|c|c|}
\hline & \multicolumn{5}{|c|}{ Points } & \multicolumn{6}{|c|}{ Patients } \\
\hline & & 1 & 2 & 3 & 4 & 5 & 6 & 7 & 8 & 9 & 10 \\
\hline \multicolumn{12}{|l|}{ T-Stage } \\
\hline $\mathrm{T} 1$ & 58 & & $\sqrt{ }$ & & & & & & $\sqrt{ }$ & $\sqrt{ }$ & \\
\hline $\mathrm{T} 2$ & 30 & & & & $\sqrt{ }$ & & $\sqrt{ }$ & & & & $\sqrt{ }$ \\
\hline $\mathrm{T} 3$ & 20 & & & $\sqrt{ }$ & & $\sqrt{ }$ & & $\sqrt{ }$ & & & \\
\hline $\mathrm{T} 4$ & 0 & $\sqrt{ }$ & & & & & & & & & \\
\hline \multicolumn{12}{|l|}{ N-Stage } \\
\hline No & 0 & & & $\sqrt{ }$ & & & $\sqrt{ }$ & & $\sqrt{ }$ & $\sqrt{ }$ & $\sqrt{ }$ \\
\hline N1 & 12.5 & & & & $\sqrt{ }$ & $\sqrt{ }$ & & & & & \\
\hline N2 & 46 & $\sqrt{ }$ & $\sqrt{ }$ & & & & & $\sqrt{ }$ & & & \\
\hline N3 & 87 & & & & & & & & & & \\
\hline \multicolumn{12}{|l|}{ ER } \\
\hline+ & 0 & & $\sqrt{ }$ & & $\sqrt{ }$ & $\sqrt{ }$ & $\sqrt{ }$ & $\sqrt{ }$ & $\sqrt{ }$ & & $\sqrt{ }$ \\
\hline- & 30 & $\sqrt{ }$ & & $\sqrt{ }$ & & & & & & $\sqrt{ }$ & \\
\hline \multicolumn{12}{|l|}{$P R$} \\
\hline+ & 0 & & $\sqrt{ }$ & & $\sqrt{ }$ & $\sqrt{ }$ & $\sqrt{ }$ & $\sqrt{ }$ & $\sqrt{ }$ & & $\sqrt{ }$ \\
\hline- & 27.5 & $\sqrt{ }$ & & $\sqrt{ }$ & & & & & & $\sqrt{ }$ & \\
\hline \multicolumn{12}{|l|}{ Histology } \\
\hline CIS-Mi 100 & & & & & & & $\sqrt{ }$ & & & & \\
\hline IDC & 94 & $\sqrt{ }$ & $\sqrt{ }$ & $\sqrt{ }$ & $\sqrt{ }$ & $\sqrt{ }$ & & $\sqrt{ }$ & & $\sqrt{ }$ & \\
\hline ILC & 20 & & & & & & & & $\sqrt{ }$ & & $\sqrt{ }$ \\
\hline \multicolumn{12}{|l|}{ Others 0} \\
\hline Total points & & 197.5 & 198 & 171.5 & 136.5 & 126.5 & 130 & 160 & 78 & 209.5 & 50 \\
\hline \multicolumn{12}{|c|}{ Predictive probability of HER2 positive } \\
\hline High & & $\sqrt{ }$ & $\sqrt{ }$ & $\sqrt{ }$ & & & & $\sqrt{ }$ & & $\sqrt{ }$ & \\
\hline Low & & & & & $\sqrt{ }$ & $\sqrt{ }$ & $\sqrt{ }$ & & $\sqrt{ }$ & & $\sqrt{ }$ \\
\hline \multicolumn{12}{|c|}{ HER2 status in CNB tissue } \\
\hline+ & & $\sqrt{ }$ & $\sqrt{ }$ & $\sqrt{ }$ & & $\sqrt{ }$ & $\sqrt{ }$ & & & & \\
\hline$+/-$ & & & & & & & & & $\sqrt{ }$ & $\sqrt{ }$ & $\sqrt{ }$ \\
\hline- & & & & & $\sqrt{ }$ & & & $\sqrt{ }$ & & & \\
\hline \multicolumn{12}{|c|}{ HER2 status in excision tissue } \\
\hline+ & & $\sqrt{ }$ & $\sqrt{ }$ & $\sqrt{ }$ & & & & $\sqrt{ }$ & & $\sqrt{ }$ & \\
\hline- & & & & & $\sqrt{ }$ & $\sqrt{ }$ & $\sqrt{ }$ & & $\sqrt{ }$ & & $\sqrt{ }$ \\
\hline
\end{tabular}


the optimal cutoff value and the nomogram, we predicted HER2 status correctly in several patients of different TNM stage and HR status. Since there're many rural areas in China that do not have sufficient resources and medical insurance coverage, our prediction model has practical value. For patients who cannot afford the FISH assay and trastuzumab therapy, our model will be helpful for predicting their HER2 status. If a patient with IHC-determined HER2-borderline disease were predicted to be HER2-positive and that patient could not afford trastuzumab, a stronger chemotherapy regimen, e.g., dose-dense AC-T, could be considered as an alternative to $\mathrm{TC}$ regimen.

One major limitation of our study was that we couldn't analyze other important pathological parameters such as tumor grade and Ki67 due to incomplete information. The database consisted of breast cancer patients diagnosed during 1999 to 2008 when the pathologic diagnosis of breast cancer in China was rapidly developing and the parameters may vary in different institution at that time, so the tumor grade, Ki67 and other parameters which are proved of prognostic importance were not included in this retrospective study. Further effort is required to improving the database by adding new cases with complete information, so that we may find more risk factors for HER2 positive breast cancer and the nomogram may be adjusted to be more accurate, hence, patients could obtain more benefit from this nomogram by given the right therapy regimens.

\section{Conclusions}

The disconcordance of HER2 status between CNB and excisional biopsies of the same tumor may cause inappropriate neoadjuvant therapy in women with HER-2 positive breast cancer. Based on the nation-wide multicenter data spans 10 years in China, we found that ER negative, PR negative, T1 stage and N2/N3 stage were independent risk factors for HER2 positive breast cancer. We further establish the nomogram for HER2 status prediction which is validated both internally and externally. The nomogram could be a valuable tool for improving the accuracy of HER2 assessment pre-operatively. By combining CNB and the nomogram, clinicians could get more information of one individual patient and provide more suitable treatment accordingly.

\section{Additional file}

Additional file 1: Figure S1. Geographic distribution of sites included in the study ${ }^{1}$. Figure S2. The age distribution of breast cancer in Chinese women ${ }^{2}$. (DOCX $459 \mathrm{~kb}$ )

\section{Abbreviations}

AUC: The areas under the receiver operating characteristic curve:

Cls: Confidence intervals; CNB: Core needle biopsy; ER: Estrogen receptor;
FNA: Fine needle aspiration; HER2: Human epidermal growth factor receptor 2; HR: Hormone receptor; IDC: Invasive ductal carcinoma;

IHC: Immunohistochemical; ORs: Odds ratios; pCR: Pathologic complete response; PR: Progesterone receptor; ROC: Receiver-operating characteristic

\section{Acknowledgements}

We thank Cancer Foundation of China for originating this retrospective clinical epidemiological study of breast cancer. We thank the local investigators from Beijing, Liaoning (Shenyang), Hunan (Changsha), Guangdong (Guangzhou), Zhejiang (Hangzhou), Shaanxi (Xi'an), and Sichuan (Chengdu) for data collection and assisting us in the successful completion of the project. The authors also thank Pfizer for funding in the form of donations to Cancer Foundation of China.

\section{Funding}

Not applicable.

\section{Availability of data and materials}

The data generated during this study are included in this article and its supplementary materials. Raw data are available upon reasonable request.

\section{Authors' contributions}

Conception and design: $\mathrm{HZ}, \mathrm{JH}$ and $\mathrm{KW}$. Provision of study material or patients: $J H, R H, W F, Y K, F X, L Z, Q S, J L, B Z, J F, Y Q, X X$ and $S Z$. Collection and assembly of data: PX, ZL, RH, WF, YK, FX, LZ, QS, JL, BZ, JF, YQ, XX and SZ. Data analysis and interpretation: $\mathrm{HZ}, \mathrm{JH}$ and $\mathrm{KW}$. Manuscript writing: $\mathrm{HZ}$ and KW. Final approval of manuscript: all authors

\section{Ethics approval and consent to participate}

The study protocol was approved by the Ethical Committee of centers including the First Affiliated Hospital of Xi'an Jiaotong University, Cancer Institute \& Hospital, Chinese Academy of Medical Sciences \& Peking Union Medical College, West China School of Public Health, Sichuan University, Zhejiang Cancer Hospital, Sun Yat-Sen University Cancer Center, Xiangya Second Hospital, Central South University and Liaoning Cancer Hospital. Patient consent for this study was not required due to the retrospective nature of our analysis.

\section{Consent for publication}

Not applicable.

\section{Competing interests}

The authors declare that they have no competing interests.

\section{Publisher's Note}

Springer Nature remains neutral with regard to jurisdictional claims in published maps and institutional affiliations.

\section{Author details}

${ }^{1}$ Department of Breast Surgery, the First Affiliated Hospital of Xi'an Jiaotong University, 277 West Yanta Road, 710061 Xi'an, People's Republic of China. ${ }^{2}$ Department of Cancer Epidemiology, Cancer Institute \& Hospital, Chinese Academy of Medical Sciences \& Peking Union Medical College, Beijing, People's Republic of China. ${ }^{3}$ Department of Epidemiology, West China School of Public Health, Sichuan University, Chengdu, Sichuan, People's Republic of China. ${ }^{4}$ Department of Breast Surgery, Zhejiang Cancer Hospital, Hangzhou, People's Republic of China. ${ }^{5}$ Department of Breast Oncology, Sun Yat-Sen University Cancer Center, Guangzhou, People's Republic of China.

${ }^{6}$ Department of Breast-thyroid Surgery, Xiangya Second Hospital, Central South University, Changsha, People's Republic of China. ${ }^{7}$ Department of Breast Surgery, Liaoning Cancer Hospital, Shenyang, People's Republic of China. ${ }^{8}$ Center of Breast Disease, Cancer Institute \& Hospital, Chinese Academy of Medical Sciences \& Peking Union Medical College, Beijing, People's Republic of China. ${ }^{9}$ Department of Pathology, Cancer Institute \& Hospital, Chinese Academy of Medical Sciences \& Peking Union Medical College, Beijing, People's Republic of China. 
Received: 17 January 2019 Accepted: 27 March 2019

Published online: 04 May 2019

\section{References}

1. Bray F, Ferlay J, Soerjomataram I, Siegel RL, Torre LA, Jemal A. Global cancer statistics 2018: GLOBOCAN estimates of incidence and mortality worldwide for 36 cancers in 185 countries. CA Cancer J Clin. 2018;68(6):394-424.

2. Generali D, Ardine M, Strina C, Milani M, Cappelletti MR, Zanotti L, et al. Neoadjuvant treatment approach: the Rosetta stone for breast Cancer? J Natl Cancer Inst Monogr. 2015;2015(51):32-5.

3. Schneeweiss A, Chia S, Hickish T, Harvey V, Eniu A, Hegg R, et al. Pertuzumab plus trastuzumab in combination with standard neoadjuvant anthracycline-containing and anthracycline-free chemotherapy regimens in patients with HER2-positive early breast cancer: a randomized phase II cardiac safety study (TRYPHAENA). Ann Oncol. 2013:24(9):2278-84.

4. Gianni L, Pienkowski T, Im YH, Roman L, Tseng LM, Liu MC, et al. Efficacy and safety of neoadjuvant pertuzumab and trastuzumab in women with locally advanced, inflammatory, or early HER2-positive breast cancer (NeoSphere): a randomised multicentre, open-label, phase 2 trial. Lancet Oncol. 2012;17(6):25-32.

5. De AE, Holmes AP, Piccartgebhart M, Holmes E, Di CS, Swaby RF, et al. Lapatinib with trastuzumab for HER2-positive early breast cancer (NeoALTTO): survival outcomes of a randomised, open-label, multicentre, phase 3 trial and their association with pathological complete response. Lancet Oncol. 2014;15(10):1137-46.

6. Guarneri V, Frassoldati A, Bottini A, Cagossi K, Bisagni G, Sarti S, et al. Preoperative chemotherapy plus trastuzumab, lapatinib, or both in human epidermal growth factor receptor 2-positive operable breast cancer: results of the randomized phase II CHER-LOB study. J Clin Oncol. 2012;30(16):1989.

7. Gianni L, Pienkowski T, Im YH, Tseng LM, Liu MC, Lluch A, et al. 5-year analysis of neoadjuvant pertuzumab and trastuzumab in patients with locally advanced, inflammatory, or early-stage HER2-positive breast cancer (NeoSphere): a multicentre, open-label, phase 2 randomised trial. Lancet Oncol. 2016;17(6):791-800.

8. de Azambuja E, Holmes AP, Piccart-Gebhart M, Holmes E, Di Cosimo S, Swaby RF, et al. Lapatinib with trastuzumab for HER2-positive early breast cancer (NeoALTTO): survival outcomes of a randomised, open-label, multicentre, phase 3 trial and their association with pathological complete response. Lancet Oncol. 2014;15(10):1137-46.

9. Ough M, Velasco J, Hieken TJ. A comparative analysis of core needle biopsy and final excision for breast cancer: histology and marker expression. Am J Surg. 2011;201(5):692-4

10. Tamaki K, Sasano H, Ishida T, Miyashita M, Takeda M, Amari M, et al. Comparison of core needle biopsy (CNB) and surgical specimens for accurate preoperative evaluation of ER, PgR and HER2 status of breast cancer patients. Cancer Sci. 2010;101(9):2074-9.

11. D'Alfonso T, Liu YF, Monni S, Rosen PP, Shin SJ. Accurately assessing her-2/ neu status in needle core biopsies of breast cancer patients in the era of neoadjuvant therapy: emerging questions and considerations addressed. Am J Surg Pathol. 2010;34(4):575-81.

12. Park SY, Kim KS, Lee TG, Park SS, Kim SM, Han W, et al. The accuracy of preoperative core biopsy in determining histologic grade, hormone receptors, and human epidermal growth factor receptor 2 status in invasive breast cancer. Am J Surg. 2009;197(2):266-9.

13. Li J, Zhang B-N, Fan J-H, Pang Y, Zhang P, Wang S-L, et al. A nation-wide multicenter 10-year (1999-2008) retrospective clinical epidemiological study of female breast cancer in China. BMC Cancer. 2011;11(1):364.

14. Zhang B-L, Sivasubramaniam PG, Zhang Q, Wang J, Zhang B, Gao J-D, et al. Trends in radical surgical treatment methods for breast malignancies in China: a multicenter 10-year retrospective study. Oncologist. 2015;20(9): 1036-43.

15. Shan Z, Jing-Qiao B, Jing L, Jin-Hu F, Yi P, Qing-Kun S, et al. The pathologic characteristics of breast cancer in China and its shift during 1999-2008: national-wide multicenter cross-sectional image over 10 years. Int J Cancer 2012;131(11):2622-31.

16. Youden WJ. Index for rating diagnostic tests. Cancer. 1950;3(1):32-5.

17. Buzdar AU, Ibrahim NK, Francis D, Booser DJ, Thomas ES, Theriault RL, et al. Significantly higher pathologic complete remission rate after neoadjuvant therapy with trastuzumab, paclitaxel, and epirubicin chemotherapy: results of a randomized trial in human epidermal growth factor receptor 2-positive operable breast cancer. J Clin Oncol. 2005;23(16):3676.
18. Fishman JE, Milikowski C, Ramsinghani R, Velasquez MV, Aviram G. USguided core-needle biopsy of the breast: how many specimens are necessary? Radiology. 2003;226(3):779.

19. Melotti MK, Berg WA. Core needle breast biopsy in patients undergoing anticoagulation therapy: preliminary results. AJR Am J Roentgenol. 2000;174(1):245-9.

20. Traina A, Agostara B, Marasà L, Calabrò M, Zarcone M, Carruba G. HER2/neu expression in relation to Clinicopathologic features of breast Cancer patients. Ann N Y Acad Sci. 2006;1089(1):159.

21. Bouchbika Z, Benchakroun N, Taleb A, Jouhadi H, Tawfiq N, Sahraoui S, et al. Association between overexpression of Her-2 and other Clinicopathologic prognostic factors in breast Cancer in Morocco. J Cancer Therapy. 2012;03(5):787-92

22. Guo Q, Kai C, Lin X, Yi S, Rui X, Yan D, et al. A nomogram to predict HER2 status in breast cancer patients with HER2-borderline disease as determined via immunohistochemistry. Oncotarget. 2017;8(55):93492-501.

23. lasonos A, Schrag D, Raj GV, Panageas KS. How to build and interpret a nomogram for cancer prognosis. J Clin Oncol. 2008;26(8):1364-70.

24. Balachandran VP, Gonen M, Smith JJ, Dematteo RP. Nomograms in oncology: more than meets the eye. Lancet Oncol. 2015;16(4):e173.

\section{Ready to submit your research? Choose BMC and benefit from:}

- fast, convenient online submission

- thorough peer review by experienced researchers in your field

- rapid publication on acceptance

- support for research data, including large and complex data types

- gold Open Access which fosters wider collaboration and increased citations

- maximum visibility for your research: over $100 \mathrm{M}$ website views per year

At BMC, research is always in progress.

Learn more biomedcentral.com/submissions 